Received: 18 July 2018

Accepted: 17 October 2018

Published online: 26 November 2018

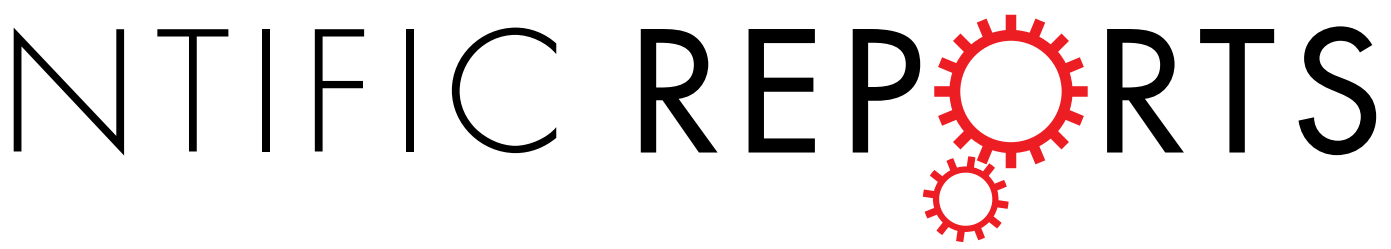

\title{
Transmission of Cricket paralysis virus via exosome-like vesicles during infection of Drosophila cells
}

\author{
Craig H. Kerr ${ }^{1,2}$, Udit Dalwadi ${ }^{1}$, Nichollas E. Scott $\mathbb{D}^{3}$, Calvin K. Yip ${ }^{1}$, Leonard J. Foster ${ }^{1,2}$ \& \\ Eric Jan $\mathbb{D}^{1}$
}

Viruses are classically characterized as being either enveloped or nonenveloped depending on the presence or absence of a lipid bi-layer surrounding their proteinaceous capsid. In recent years, many studies have challenged this view by demonstrating that some nonenveloped viruses (e.g. hepatitis A virus) can acquire an envelope during infection by hijacking host cellular pathways. In this study, we examined the role of exosome-like vesicles (ELVs) during infection of Drosophilia melanogaster S2 cells by Cricket paralysis virus (CrPV). Utilizing quantitative proteomics, we demonstrated that ELVs can be isolated from both mock- and CrPV-infected S2 cells that contain distinct set of proteins compared to the cellular proteome. Moreover, 40 proteins increased in abundance in ELVs derived from CrPV-infected cells compared to mock, suggesting specific factors associate with ELVs during infection. Interestingly, peptides from CrPV capsid proteins (ORF2) and viral RNA were detected in ELVs from infected cells. Finally, ELVs from CrPV-infected cells are infectious suggesting that CrPV may hijack ELVs to acquire an envelope during infection of S2 cells. This study further demonstrates the diverse strategies of nonenveloped viruses from invertebrates to vertebrates to acquire an envelope in order to evade the host response or facilitate transmission.

Traditionally, viruses have been categorized into one of two classes: enveloped or nonenveloped ${ }^{1}$. Envelopes are acquired through non-lytic release from the host cell whereby the virus typically 'buds' from a distinct membrane in the cell. For example, hepatitis $\mathrm{C}$ virus, hantavirus, and influenza virus bud from the ER, Golgi, and plasma membrane, respectively ${ }^{2-4}$. Envelopes provide the virus advantages including the ability for the virus to circulate and avoid immune detection. Peplomers embedded within an envelope render it indispensable for those viruses that harbor one as it provides key determinants for cell tropism and mechanism of entry into host cells. By contrast, the absence of an envelope bestows its own advantages; nonenveloped viruses are characteristically more resistant to chemical treatments and have greater environmental stability, thus allowing them to persist outside of a host for longer. Viruses belonging to this class, such as adenovirus and picornaviruses, assemble and accumulate in nonlumenal compartments until the host cell membrane is dismantled leading to release of the progeny virions. Whether a virus has evolved to possess an envelope or not has a substantial impact on how it is transmitted and recognized by the host immune system.

The classification of enveloped or nonenveloped viruses has been recently challenged by surmounting evidence demonstrating non-lytic spread of several nonenveloped viruses in both tissue culture and animal hosts ${ }^{5-8}$. Recent work demonstrated that poliovirus, coxsackievirus B3, and rhinovirus usurp the autophagic pathway to package virions en bloc into a single bilayer phosphatidylserine-containing vesicles that are non-lytically released from the host cell ${ }^{6}$. It is proposed that a double-membraned vesicle wraps the virions and then fuses with the plasma membrane, therefore releasing a virion with a single bilayer. This mode of transmission is thought to increase infection efficiency and may permit genetic complementation between quasi-species. In contrast to poliovirus and CVB3, hepatitis A virus (HAV), another member of the picornaviridae, hijacks the exosomal pathway to acquire an envelope for non-lytic release ${ }^{5}$. HAV interacts with ALIX to use the ESCRT-III complex to drive sorting of HAV virions into multivesicular bodies (MVBs), thus leading to secretion of membranous virions from the host cell. The envelope

${ }^{1}$ Department of Biochemistry and Molecular Biology, Life Sciences Institute, University of British Columbia, Vancouver BC, V6T 1Z3, Canada. ${ }^{2}$ Michael Smith Laboratories, University of British Columbia, Vancouver BC, V6T 1Z3, Melbourne, Australia. ${ }^{3}$ Department of Microbiology and Immunology, University of Melbourne, Melbourne, Australia. Correspondence and requests for materials should be addressed to L.J.F. (email: foster@msl.ubc.ca) or E.J. (email: ej@mail.ubc.ca) 
acquired by HAV offers the advantage of cloaking it from neutralizing antibodies that circulate in the host blood leaving the virus able to spread from cell-to-cell. Nonenveloped HAV virions are found in the stool of infected chimpanzees, suggesting that the acquisition of an envelope is not a passive mechanism and may be cell type specific ${ }^{5}$.

In general, exosomes are small $(30-100 \mathrm{~nm})$ vesicles of endocytic origin that are secreted by all mammalian cell types. They have been shown to contain active enzymes, metabolites, mRNAs, and small RNAs that are transported to adjacent cells ${ }^{9,10}$ and thus, exosomes are thought to participate in cell-to-cell communication. Exosomes play a significant role during viral infection by facilitating both the host immune response and viral pathogenesis $^{11,12}$. Interestingly, exosome-like vesicles are also secreted by virus-infected insect cells, suggesting an ancestral cell-to-cell communication pathway ${ }^{13-15}$. A recent report demonstrated that exosome-like vesicles are secreted by haemocytes in virus-infected fruit flies that contain virally-derived siRNAs, which are then delivered to cells throughout the fly eliciting a long-lasting systemic RNAi respons ${ }^{16}$. Still, little is known if insect viruses, much like their mammalian counterparts, can utilize extracellular vesicles to their own advantage.

Here, we investigate the role of exosome-like vesicles in Drosophila S2 cells during cricket paralysis virus $(\mathrm{CrPV})$ infection. CrPV is a member of the dicistroviridae family that includes Drosophila $\mathrm{C}$ virus and Israeli acute paralysis virus. Dicistroviruses have served as a model for understanding virus host interactions in invertebrates such as viral translational controls and antiviral responses ${ }^{17,18}$. CrPV is a positive-strand RNA virus $\sim 9 \mathrm{~kb}$ in length that encodes two main open reading frames. Translation of the CrPV ORFs is mediated by distinct internal ribosome entry sites (IRES); the $5^{\prime}$ untranslated region ( $5^{\prime} \mathrm{UTR}$ ) IRES directs the first open reading frame (ORF1) which encodes the viral non-structural proteins such as the RNA-dependent RNA polymerase (RdRp) and the 3C-like protease and the intergenic (IGR) IRES drives ORF2 which encodes the viral structural proteins ${ }^{18}$. The life cycle of CrPV infection in S2 cells is relatively fast; after virus adsorption, CrPV infection leads to cytopathic effects by 10-12 hours post infection (hpi). Egress of CrPV is thought to primarily to occur via lytic release; however, it has not been examined in detail whether alternative egress paths exist. In this study, we examined the proteomes of ELVs from mock- and CrPV-infected cells and reveal that CrPV structural proteins are present in ELVs. We demonstrate that CrPV, in addition to lytic release, appears to hijack exosome-like vesicles for non-lytic release to promote viral infection within Drosophila cells.

\section{Materials and Methods}

Cell culture and virus. Drosophila Schneider line 2 (S2) cells were maintained and passaged in Shield's and Sang medium (Sigma) supplemented with $10 \%$ fetal bovine serum depleted in exosomes at $25^{\circ} \mathrm{C}$. Exosomes were depleted by spinning FBS at 120,000 relative centrifugal force (RCF) for $18 \mathrm{~h}$ at $4{ }^{\circ} \mathrm{C}$. Propagation and purification of CrPV in Drosophila S2 cells has been previously described ${ }^{19}$.

Viral titres were determined as previously described ${ }^{19}$. Briefly, a total of $1.5 \times 10^{6} \mathrm{~S} 2$ cells we incubated with serial dilutions of infected-cell supernatant for $30 \mathrm{~min}$, resuspended in media, and added into a 96 -well plate coated with concavilin A $\left(0.5 \mathrm{mg} / \mathrm{mL}\right.$; Calbiochem). Plates were incubated at $25^{\circ} \mathrm{C}$ for $8 \mathrm{~h}$. Cells were then washed with PBS before fixation with 3\% paraformaldehyde for $15 \mathrm{~min}$ followed by methanol permeabilization for $10 \mathrm{~min}$. Fixed cells were incubated with an anti-ORF2 antibody (1:250 dilution in 5\% bovine serum albumin in PBS) for $1 \mathrm{~h}$ at room temperature. Subsequently, cells were washed three times with PBS and incubated with a Texas Red IgG anti-rabbit (1:500 dilution in 5\% bovine serum albumin in PBS; Invitrogen) for $1 \mathrm{~h}$ at room temperature. Finally, cells were washed with PBS and stained with Hoechst dye $(0.5 \mu \mathrm{g} / \mathrm{mL})$. Plates were analyzed with a Cellomics Arrayscan HCS instrument and the number of infected cells was used to determine viral titres. Each titre is the result of at least three replicate experiments.

Cells infections with CrPV. For all infections involving CrPV, S2 cells were first pelleted at 200 RCF for $5 \mathrm{~min}$ before being resuspended in $100 \mathrm{uL}$ of PBS containing a corresponding amount of virus (e.g. for MOI 10, $1.0 \times 10^{6}$ cells were infected with $1.0 \times 10^{7} \mathrm{FFU}$ of $\mathrm{CrPV}$ ). $\mathrm{CrPV}$ was allowed to adsorb for $30 \mathrm{~min}$ at $25^{\circ} \mathrm{C}$ with agitation. Subsequently, cells were washed twice with 1X PBS, pelleted, resuspended in exosome-depleted media, and incubated at $25^{\circ} \mathrm{C}$.

Exosome isolation. Exosomes were isolated from S2 cells as previously described ${ }^{13} .5 .0 \times 10^{7}$ cells were cultured in $50 \mathrm{~mL}$ of exosome-depleted media for $24 \mathrm{~h}$. Cells and media were harvested and cells were pelleted at 300 RCF for $5 \mathrm{~min}$. Cells were kept for further analysis downstream. Media was cleared of cellular debris by serial centrifugation at $5000 \mathrm{RCF}$ for $10 \mathrm{~min}$ followed by $10,000 \mathrm{RCF}$ for $30 \mathrm{~min}$ both at $4{ }^{\circ} \mathrm{C}$. The supernatant was then under laid with a $5 \mathrm{~mL} 45 \%$ sucrose cushion and ultracentrifuged at $100,000 \mathrm{RCF}$ for $2 \mathrm{~h}$ at $4^{\circ} \mathrm{C}$. The interphase containing the exosomes was kept by removing $45 \mathrm{~mL}$ of the supernatant and $2.5 \mathrm{~mL}$ of the sucrose cushion. Exosomes were then pelleted by diluting the interphase with $50 \mathrm{~mL}$ of $1 \mathrm{X}$ PBS and ultracentrifuging at $100,000 \mathrm{RCF}$ for $2 \mathrm{~h}$ at $4{ }^{\circ} \mathrm{C}$.

For CrPV derived exosomes used in sucrose gradients, Western blot analysis, and proteomic analysis, S2 cells were infected with $\mathrm{CrPV}$ for $6 \mathrm{~h}$ (MOI 10) by washing the cells with PBS and then incubating with virus at $25^{\circ} \mathrm{C}$ for 30 mins. After viral adsorption, cells were washed with PBS followed by addition of exosome-depleted media and incubation at $25^{\circ} \mathrm{C}$. The supernatant was harvested and exosomes were isolated as stated above. For analysis of enveloped CrPV, S2 cells were infected for $24 \mathrm{~h}$ (MOI 10) before harvesting the supernatant.

Sucrose and iodixanol gradients. For density estimation of exosomes from S2 cells, exosomes were first isolated as above and then layered onto an $11 \mathrm{~mL}$ linear sucrose gradient $(0.25-2 \mathrm{M}$ sucrose). The gradients were then centrifuged at $100,000 \mathrm{RCF}$ for $18 \mathrm{~h}$ at $4{ }^{\circ} \mathrm{C}$. Fractions were collected using an ISCO fractionator and density was determined by comparing fractions to a standard curve using a Brix/RI-Check refractometer (Reichart).

To separate exosomes and enveloped CrPV from non-enveloped virus we adapted a protocol that employs isopycnic iodixanol, or Optiprep, gradients (Invitrogen) to separate enveloped from non-enveloped virus ${ }^{5}$. Briefly, 
cell-culture supernatants were clarified by serial centrifugation at $1000 \mathrm{RCF}$ for 10 minutes at $4{ }^{\circ} \mathrm{C}$, followed by $10,000 \mathrm{RCF}$ for 30 minutes at $4^{\circ} \mathrm{C}$. Virus and exosomes were then pelleted by centrifuging at 100,000 RCF for $1 \mathrm{~h}$ at $4{ }^{\circ} \mathrm{C}$. The resulting pellet was resuspended in $500 \mu \mathrm{L}$ of $1 \mathrm{X}$ PBS and layered onto an $8-40 \%$ iodixanol step gradient. Gradients were centrifuged at 141,000 RCF in a SW 41 Ti rotor (Beckman) for $48 \mathrm{~h}$ at $4{ }^{\circ} \mathrm{C}$. Fractions were collected from the top by hand.

Protein digestion and duplex demethylation labeling. Cell or exosome pellets harvested from $\mathrm{CrPV}$-infected cells at $6 \mathrm{hpi}$ were solubilized in $6 \mathrm{M}$ urea and $2 \mathrm{M}$ thiourea. Protein concentrations were determined via BCA assay (Thermo). Equal amounts of protein $(50 \mu \mathrm{g})$ from each sample was reduced by adding $1 \mu \mathrm{g}$ of dithiothretiol and incubating for $30 \mathrm{~min}$ at room temperature. Proteins were alkylated with the addition of $5 \mu \mathrm{g}$ iodoacetamide and allowed to incubate for $20 \mathrm{~min}$ at room temperature. Samples were digested with LysC before dilution with 4 volumes of $50 \mathrm{mM}$ ammonium bicarbonate and digestion overnight with trypsin. Digested peptides were purified and concentrated on C18 STAGE-tips, eluted in $80 \%$ acetonitrile, $0.5 \%$ acetic acid, and dried in a vacuum concentrator (Eppendorf). Dried peptides were resuspended in $100 \mathrm{mM}$ triethylammonium bicarbonate and chemical demethylation labeling was performed using light $\left(\mathrm{CH}_{2} \mathrm{O}\right.$; mock-infected samples) or heavy $\left({ }^{13} \mathrm{CD}_{2} \mathrm{O}\right.$; CrPV-infected samples) isotopologues of formaldehyde ${ }^{20}$. Labeled samples were combined and STAGE-tip purified. Eluted samples were dried and resuspended in $20 \%$ acetonitrile and $0.1 \%$ formic acid.

Liquid chromatography tandem mass spectrometry (LC-MS/MS) analysis. Digested peptides were analyzed by LC-MS/MS using a nanoflow HPLC (Thermo easy-nLC1000) coupled to a Q-Exactive mass spectrometer (Thermo). For each sample, $5.0 \mu \mathrm{g}$ of peptides (based on the protein quantitation) were injected into the LC and loaded onto an in-house packed fused-silica ( $5 \mu \mathrm{m}$ Aqua C18 particles (Phenomenex)) fritted trap column $(2 \mathrm{~cm}, 100 \mu \mathrm{m}$ I.D., $360 \mu \mathrm{m}$ O.D., $5 \mu \mathrm{L} / \mathrm{min}$ flow rate, Buffer $\mathrm{A}=0.5 \%$ acetic acid), then resolved on a reverse phase $75 \mu \mathrm{m}$ inner diameter fused silica, in-house packed $30 \mathrm{~cm}$ analytical column (ReproSil C18, $3 \mu \mathrm{m}$ particle size (Dr. Maisch)) using a $75 \mathrm{~min}$ linear gradient run at $250 \mu \mathrm{L} / \mathrm{min}$ from $5 \%$ to $35 \%$ Buffer B (acetonitrile, $0.5 \%$ acetic acid), followed by a $15 \mathrm{~min}$ wash at $95 \%$ Buffer B. Instrument acquisition parameters included a $1 \%$ underfill ratio, 70000 precursor mass resolution, 17500 fragment mass resolution, normalized collision energy (NCE) of 28\%, +1 and unassigned charges were excluded, "exclude isotopes" was turned on, intensity-dependent MS/MS at 1.7e5 intensity threshold, and the instrument was set to scan from 300 to $2000 \mathrm{~m} / \mathrm{z}$ with a $30 \mathrm{~s}$ dynamic exclusion time.

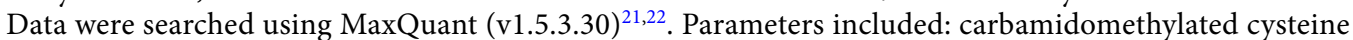
(fixed), methionine oxidation (variable), glutamine and asparagine deamidation (variable); trypsin specific; maximum 2 missed cleavages; 10 ppm precursor mass tolerance; $0.05 \mathrm{Da}$ fragment mass tolerance; $1 \%$ FDR; +1 to +7 charge states; match between runs and re-quantify; and common contaminants were included. Protein groups required a minimum of 1 peptide to be identified and a minimum of 2 peptides for quantification. Both the Drosophila and CrPV protein databases used were downloaded from Uniprot (www.uniprot.org; April 23 ${ }^{\text {rd }}$, 2014). Using Perseus (v1.5.2.6) ${ }^{23}$, contaminants and reverse hits were filtered out, protein ratios were $\log _{2}$ transformed, and ratios were averaged between replicates.

For GO term enrichment analysis, we employed the Gene Score Resampling (GSR) in ErmineJ v3.02, using the Log2 transformed protein ratios for "protein score" 24 . We considered a GO term to be significantly enriched if the Bejamini Hochberg-corrected GSR p-value was less than 0.05. The mass spectrometry proteomics data have been deposited to the ProteomeXchange Consortium via the PRIDE ${ }^{1}$ partner repository with the dataset identifier PXD011271.

RT-PCR. RNA from whole cells was extracted using TriZol reagent (Invitrogen). Alternatively, RNA was isolated from gradient fractions using phenol:chloroform extraction. Briefly, each fraction was brought up to $250 \mu \mathrm{L}$ with RNase-free $\mathrm{H}_{2} \mathrm{O}$. An equal amount of phenol was then added to each fraction, samples were vortex and centrifuged at $4^{\circ} \mathrm{C}$ at $13.2 \mathrm{~K}$ RPM for 30 minutes. The aqueous layer was removed and placed into a new tube with and equal volume of chloroform. The sample was vortexed and centrifuged for 5 minutes at $4{ }^{\circ} \mathrm{C}$ at $13.2 \mathrm{~K}$ RPM. The aqueous layer was once again removed and an equal volume of $100 \%$ ispropanol was added in addition to $400 \mathrm{mM}$ of ammonium acetate. Samples were incubated at $-20^{\circ} \mathrm{C}$ for at least $2 \mathrm{~h}$. RNA was then pelleted by centrifugation for 10 minutes at $13.2 \mathrm{~K} \mathrm{RPM}$. The RNA pellet was washed 3 times with $75 \%$ ethanol before resuspending in $10 \mu \mathrm{L}$ RNase-free $\mathrm{H}_{2} \mathrm{O}$.

For RT-PCR on gradient fractions, the entirety of the extracted RNA was used and $100 \mathrm{ng}$ of in vitro synthesized firefly luciferase RNA was added as a standard to each fraction. RT-PCR was performed using Superscript Reverse Transcriptase III (Invitrogen) at $50^{\circ} \mathrm{C}$ using a random hexamer primer. CrPV cDNA was amplified using primers P1 ( $5^{\prime}$ - TCCTCAAGCCATGTGTATAGGA - $\left.3^{\prime}\right)$ and P2 (5' - GTGGCTGAAATACTATCTCTGG - 3') while FLuc cDNA was amplified using primers P3 (5' - ATGAACGTGAATTGCTCAAC - 3') and P4 $\left(5^{\prime}-\right.$ CCGGATTGTTTACATAACC - 3').

Western blots. For cell and exosomal lysates, equal amounts of protein $(10 \mu \mathrm{g})$ were resolved on a $12 \%$ SDS-PAGE gel and then transferred to a polyvinylidene difluoride Immobilon-FL membrane (Millipore). Membranes were blocked for $30 \mathrm{~min}$ at room temperature with 5\% skim milk in TBST. Blots were incubated for $1 \mathrm{~h}$ at room temperature with the following antibodies: CrPV ORF1 (raised against CrPV RdRp) rabbit polyclonal (1:10,000), CrPV ORF2 (raised against CrPV VP2) rabbit polyclonal (1:10,000), mouse anti-syntaxin $1 \mathrm{~A}$ (1:5000; DSHB), mouse anti-ubiquitin (1:5000; AbCam), or mouse anti-Actin (1:5000; AbCam). Membranes were washed 3 times with TBST and incubated with either goat anti-rabbit IgG HRP (1:20,000; GE Healthcare) or goat anti-mouse IgG HRP (1:5000; Santa Cruz) for $1 \mathrm{~h}$ at room temperature. Enhanced chemiluminescence (Thermo) with was used for detection. 
For westerns performed on gradient fractions, proteins were first extracted using trichloroacetic acid (TCA). To each fraction an equal amount of $\mathrm{H}_{2} \mathrm{O}$ was added for a total volume of $400 \mu \mathrm{L} .100 \mu \mathrm{L}$ of $100 \%$ TCA was added and samples were incubated on ice for 10 minutes. Protein was pelleted by centrifugation for 10 minutes at $4{ }^{\circ} \mathrm{C}$ at 16.1 RCF. Supernatant was removed and the pellet was washed twice with $200 \mu \mathrm{L}$ of ice cold acetone. The final pellet was then dried by incubating at $95^{\circ} \mathrm{C}$ for 5 minutes and protein was resuspended in $20 \mu \mathrm{L}$ of SDS-PAGE loading buffer.

Electron microscopy. Negative stained specimens were prepared by adsorbing samples to glow discharged carbon-coated copper grids and subsequently staining with uranyl formate. A Tecnai Spirit transmission electron microscope (FEI) operated at an accelerating voltage of $120 \mathrm{kV}$ was used to examine these specimens. Images were acquired using a $4 \mathrm{~K} \times 4 \mathrm{~K}$ Eagle charge-coupled device (CCD) camera (FEI) at a nominal magnification of $49,000 \times$.

Membrane integrity assay. S2 cells $\left(2.5 \times 10^{6}\right)$ were seeded onto 6-well plates. Cells were washed with PBS and infected with CrPV at an MOI of 10 by incubating at $25^{\circ} \mathrm{C}$ for $30 \mathrm{~min}$. Cells were then washed with PBS before adding complete Shields and Sang media. At each time point $(2,4,6,8 \mathrm{~h})$ cells were harvested and $10 \mu \mathrm{L}$ of the cell suspension was incubated with an equal volume of trypan blue. Membrane permeability was assessed via trypan blue exclusion using a Countess II Automated Cell Counter (Thermo Fisher). In parallel, the supernatant from each time point was used for viral titre determination as described above. Light micrographs of cells were acquired using a Olympus CKX41 microscope at 40X magnification mounted with a Olympus SC30 camera.

Treatment of cells with ELVs. CrPV and ELVs were first separated via iodixanol gradients as described above. Fractions corresponding to either ELVs or CrPV virions were pooled and dialyzed in PBS using a Slide-A-Lyzer 10,000 MWCO dialysis cassette (Thermo Fisher). After dialysis, pooled fraction sets were added to $\mathrm{S} 2$ cells seeded in a 6-well plate without media and incubated for $1 \mathrm{~h}$ at $25^{\circ} \mathrm{C}$. Following incubation, cells were washed with PBS and complete S2 cell media was added. Cells were incubated at $25^{\circ} \mathrm{C}$ for $48 \mathrm{~h}$ before being harvested for viral yield determination.

\section{Results}

Isolation of exosome-like vesicles from $\mathbf{S 2}$ cells during CrPV infection. To characterize exosomelike vesicles (ELVs) under CrPV infection, we isolated ELVs from Drosophila S2 cells that were mock- or CrPVinfected using differential ultracentrifugation as previously described ${ }^{13}$. Both the S2 whole cell pellet and the ELV pellet were analyzed by Western blot to assess the presence of exosomal protein markers (Fig. 1A) ${ }^{25}$. As reported previously ${ }^{13}$, both pellets contained $\beta$-actin, and ubiquitin. Ubiquitin is thought to aid in protein sorting to exosomes on the cytosolic side of the multi-vesicular body, therefore an enrichment of specific proteins would be expected in the ELV pellet ${ }^{26}$. Using an anti-ubiquitin antibody, differential banding patterns were observed between the cell pellet and ELV fraction. (Fig. 1A). Moreover, syntaxin-1A showed a strong enrichment in the ELV fraction compared to the cell pellet as observed previously (Fig. 1A) ${ }^{13,25}$. In addition to Western blot analysis, we employed negative stain electron microscopy to assess the integrity of the vesicles. Electron micrographs of the vesicles displayed a range in size of $30-100 \mathrm{~nm}$, typical of exosomes isolated from mammalian cells (Fig. 1B) ${ }^{27}$. As reported ${ }^{13,25}$, these results indicate that bona fide exosome-like vesicles can be isolated from $\mathrm{S} 2$ cells.

We next investigated ELVs from S2 cells infected with CrPV. We chose to infect cells for $6 \mathrm{~h}$ to mitigate contamination of the ELVs by cellular debris due to lysis ${ }^{28,29}$. Similar to ELVs from mock-infected cells, syntaxin-1A was enriched in the ELV fraction compared to the cell pellet (Fig. 2). Interestingly, distinct ubiquitylated proteins could be detected when comparing ELVs isolated from mock and CrPV-infected cells suggesting that there may be changes in protein content and/or ubiquitylation of proteins in the vesicles (Fig. 2A). Using an antibody that recognizes the RNA-dependent RNA polymerase (RdRP) of CrPV, CrPV RdRP was present in the CrPV-infected cell pellets, but not in the ELV fractions suggesting that it is not packaged into ELVs.

To further validate that exosome-like vesicles were isolated, we measured their density via sucrose gradients. ELV pellets were isolated and then layered onto a $0.25-2 \mathrm{M}$ sucrose density gradient. After centrifugation, the gradients were fractionated and the distribution of syntaxin-1A across the gradient was monitored by immunoblotting (Fig. 2B). From both mock- or CrPV-infected cells, syntaxin-1A was present in fractions of a density range from $1.09-1.16 \mathrm{~g} / \mathrm{mL}$, consistent with the range of ELVs ${ }^{9}$ (Fig. 2B). Altogether these results demonstrate that exosome-like vesicles were isolated from CrPV-infected cells.

Proteomic analyses of CrPV-infected cells and derivative ELVs. Our Western blot analyses hinted that the protein composition of ELVs is different between mock- and CrPV-infected S2 cells. To address this, we utilized quantitative mass spectrometry to identify changes in the proteomes of ELVs (Fig. S4). Proteins of ELVs from mock- or CrPV-infected S2 cells $(6 \mathrm{~h})$ were isolated and digested with trypsin to produce peptides that were subsequently labeled with a 'light' formaldehyde reagent (mock-infected) or 'heavy' formaldehyde reagent (CrPV-infected) before analyzing via LC-MS/MS (Fig. S4) ${ }^{20}$. This analysis was also performed on cell pellets in parallel (with the same labeling procedure) to determine if proteins enriched in ELVs was due to an increased abundance within the cell or specific packaging of host proteins. From this analysis, we identified and quantified 1428 and 802 proteins from the cell and ELV pellets, respectively, in 2 of 3 biological replicates (Fig. 3). Of these, 913 and 287 proteins were specific to the cell and ELV pellets, respectively, while 515 proteins were shared between both (Fig. 3A). For proteins quantified in the cell pellet, only 3 displayed a $>2$-fold increase in protein abundance in CrPV-infected compared to mock-infected cells (red points; Fig. 3B). These included both viral polyproteins (ORF1 and ORF2) and the uncharacterized protein, CG31731-RC (Fig. 3B and D). The majority of proteins quantified in the cell pellet at 6 hpi decreased in protein abundance with 16 proteins being down-regulated more than 2 -fold compared to mock-infected cells (blue points; Fig. 3B). These results 
A)
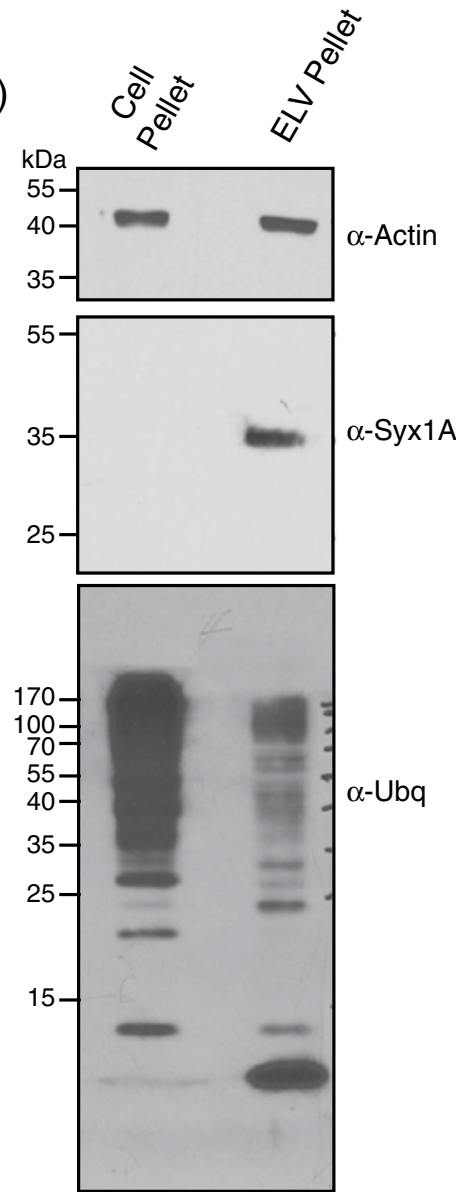

B)

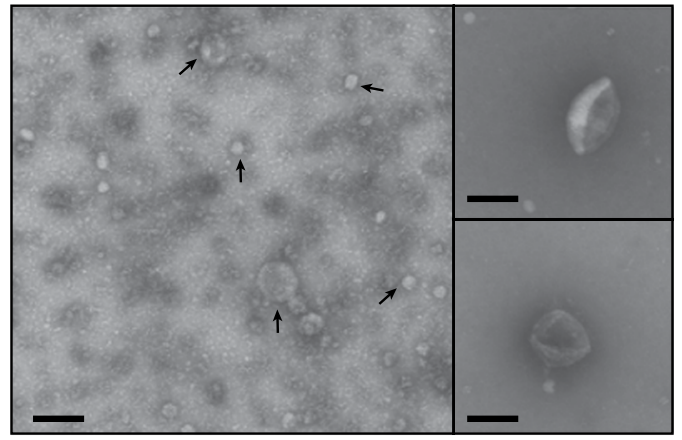

Figure 1. Validation of exosome-like vesicles isolated from Drosophila S2 cells. (A) Western blot analysis of ELV markers in the cell pellet and ELV pellet after isolation. Syx1 A = Syntaxin-1A; Ubq= Ubiquitin. (B) Transmission electron micrograph of ELVs isolated using differential ultracentrifugation. Micrographs shown are from a 1:2 dilution (left panel) or 1:50 dilution (right panels) in PBS before TEM. Scale bar represent $100 \mathrm{~nm}$. Uncropped gel images are shown in Supplemental Fig. 1.

are consistent with the observation that overall host translation is shut down in CrPV-infected cells concomitant with increased viral protein synthesis ${ }^{19,30}$. In the ELV pellet, 40 proteins increased $>2$-fold in abundance at 6 hpi while 8 proteins decreased $>2$-fold (red and blue points, respectively; Fig. $3 \mathrm{C}$ ).

We also identified multiple markers that have been associated with exosomes in the ELV pellet such as ALIX, Syntaxin-1A, Rab35, and Flotillin-1, further supporting that bona fide exosomes were isolated ${ }^{13}$. Surprisingly, CrPV ORF2 peptides were found in the ELV pellet $\left(\log _{2}\right.$ ratio $=12.04$; Figs $3 \mathrm{D}$ and 4 , and Table S3). In contrast, there were no peptides mapped to the ORF1 of CrPV in the ELV pellet; congruent with our previous Western blot analysis (Figs 2, 3D, and Tables S1 and S2).

To gain further insight into the cellular processes underlying proteins in the cell pellet and ELVs in CrPV-infected cells, we performed a gene score resampling (GSR) analysis. Unsurprisingly, there were no significantly enriched terms found within the cell pellet protein list since CrPV infection results in global translation shut down. On the other hand, many GO terms were significantly enriched (Benjimini Hochberg-corrected p-value $<0.05$ ) in ELVs, such as those linked to transport (e.g. GO:0006818; GO:0006811; GO:0055085) and metabolic processes (e.g. GO:0006091; GO:0006793; GO:0051186) (Table S1). Given that biogenesis of ELVs occurs through invagination of the endosomal membrane, it is unsurprising that a large portion of proteins are annotated with metabolic functions as these processes largely occur in the cytoplasm. Altogether, these results suggest that the protein composition of ELVs during CrPV-infection is unique compared to that of the cell pellet and ELVs derived from mock-infected cells.

CrPV may hijack ELVs during infection. The observation that ORF2 (i.e. structural), but not ORF1 proteins from $\mathrm{CrPV}$ are found in ELVs suggests that CrPV may commandeer these vesicles during infection. To rule out that the resulting CrPV-containing ELVs are a result of apoptosis/lysis from infected S2 cells, we examined the extracellular viral titres and integrity of the cell membrane by trypan blue exclusion assay over the course of an $8 \mathrm{~h}$ viral infection (Fig. 5A). Increasing extracellular virus is detected throughout the course of infection with an increase at $6 \mathrm{hpi}$ and increases substantially onwards (Fig. 5A). By $8 \mathrm{hpi}$, ten times more virus is detected in the supernatant relative to that at $2 \mathrm{hpi}$ (Fig. 5A). Interestingly, the membrane of S2 cells remains intact up until 12 
A)
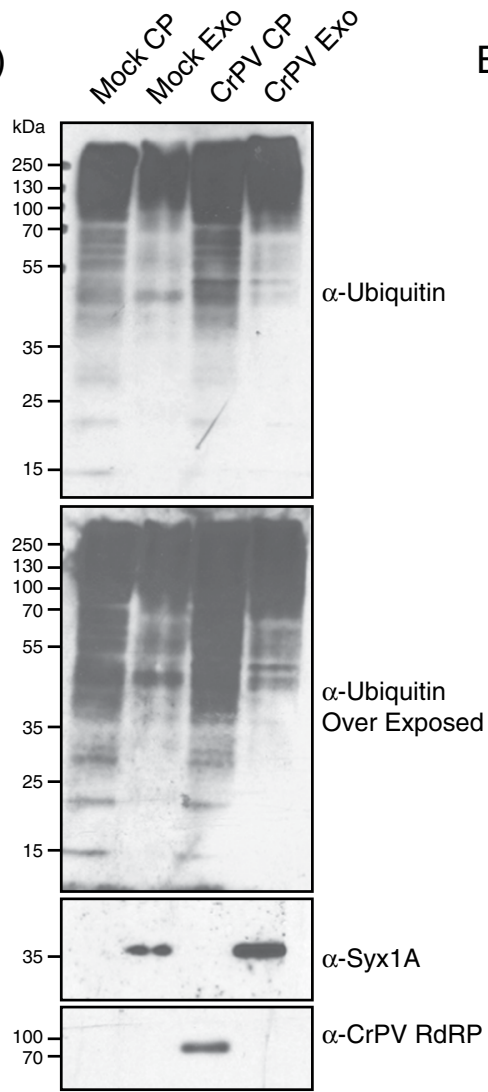

B)

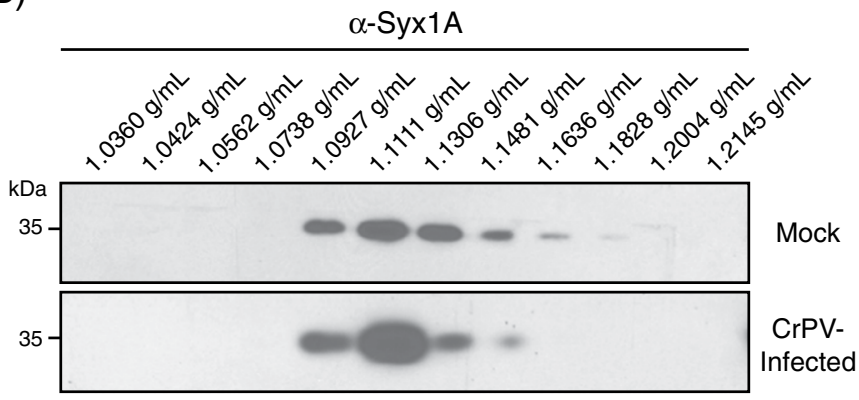

Figure 2. Isolation of Exosome-like vesicles from Drosophila S2 cells during CrPV infection. (A) Western blot analysis of the cell pellet (CP) versus the ELV pellet (Exo) in either mock- or CrPV-infected S2 cells. Cells were infected at an MOI of 10 and exosomes were harvested at 6 hours post infection. Syx $1 \mathrm{~A}=$ Syntaxin-1A; Ubq $=$ Ubiquitin. (B) Sucrose density gradient separation of exosome-like vesicles isolated from mock- or CrPV-infected cells at 6 hpi. Uncropped gel images are shown in Supplemental Figs 2, 3.

hpi, suggesting that the virus being released into the supernatant is not due to overt cell lysis. This is corroborated through examination of infected cells under light microscopy (Fig. S6). Minimal cytopathic effects are visible (i.e. lifting off the substratum) up until $10 \mathrm{hpi}$ and onwards where distinct cell lysis is observed (Fig. S6; black arrows).

It is possible that CrPV virions are associated with the ELVs and not necessarily within the vesicles. To distinguish these possibilities, we performed iodixanol gradients on the extracellular contents of CrPV-infected S2 cells. Iodixanol gradients are sensitive to changes in sample densities and thus exosomes and virions fractionate differentially $^{31}$. From CrPV-infected S2 cells, supernatants were collected and ELVs (and virus) were pelleted via ultracentrifugation before layering onto an $8-40 \%$ iodixanol gradient. The gradient was subsequently fractionated and analyzed by Western blot and RT-PCR analyses for the presence of viral proteins or RNA, respectively. Consistent with our mass spectrometry data, CrPV VP2 structural protein had a bimodal distribution, with one population that co-fractionates with syntaxin-1A, which represents ELVs and one that sediments to the bottom of the gradient (Fig. 5B), the latter of which likely represents fully assembled virions containing viral RNA. For RT-PCR analysis, equal amounts of a control in vitro transcribed luciferase RNA were aliquoted into each fraction, which was used as a RT-PCR normalization control across the gradient. The CrPV RNA exhibited a bimodal distribution that co-sedimented similarly to the VP2 protein, thus suggesting that both viral RNA and protein are within the ELV fractions. To determine if the syntaxin-1A-containing fractions include virions enveloped with a lipid, we treated isolated ELV pellets with membrane-disrupting NP-40 detergent before layering onto the iodixanol gradients. Comparing to untreated ELVs, NP-40-treated ELVs resulted in a broad distribution of syntaxin $1 \mathrm{~A}$, indicating that the lipid envelope of ELVs was disrupted. Furthermore, NP-40 treatment abolished the bimodal distribution of CrPV VP2 and RNA resulting in an enriched population near the bottom of the gradient, strongly suggesting that the CrPV structural proteins and RNA are enveloped with a membrane within ELVs (Fig. 5B).

We next determined whether the light and dense fractions containing CrPV protein/RNA are infectious. Pooled fractions containing 'naked' CrPV (fractions 15-17) or 'enveloped' CrPV (eCrPV; fractions 5-9) were dialyzed with PBS and then incubated with S2 cells for $48 \mathrm{~h}$ (Fig. 6A). Supernatants from cells incubated with either $\mathrm{CrPV}$ or eCrPV resulted in an approximately equal amount of viral titre after $48 \mathrm{~h}$, suggesting that both CrPV and eCrPV fractions are infectious (Fig. 6). Taken together, these data suggest that CrPV can commandeer ELVs to facilitate infection in Drosophila cells. 


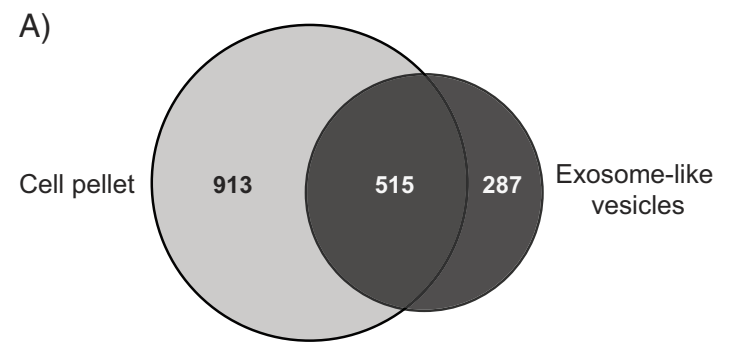

B)

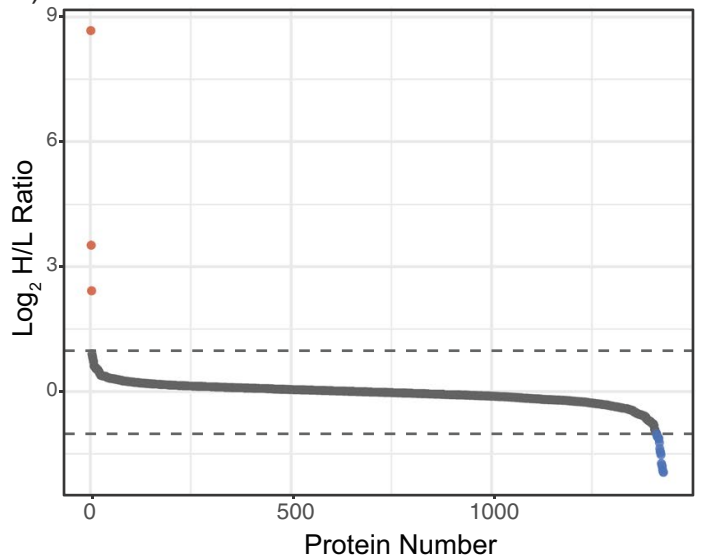

C)

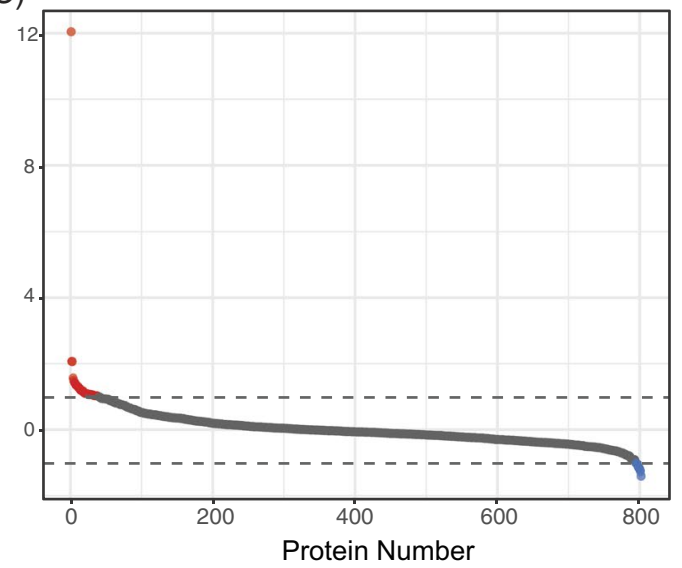

D)

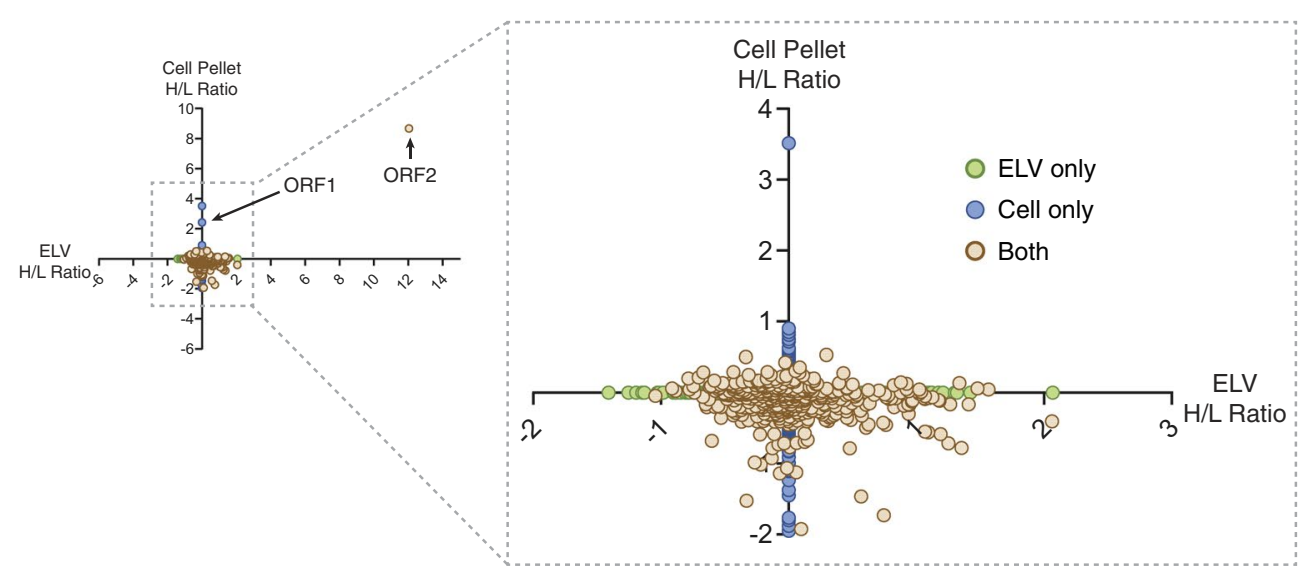

Figure 3. Quantitative proteomic comparison of cell pellets and ELVs isolated from mock- and CrPV-infected cells at 6 hours post infection. (A) Venn diagram of proteins identified in S2 cell pellets and ELVs from both mock- and CrPV-infected cells. Cells and ELVs were collected from both mock and CrPV-infected S2 cells at 6 hpi. Proteins were collected from all samples and equal amounts were subjected to trypsin digestion. Peptides from both the cell pellet and ELV pellets were dimethylated labeled with light formaldehyde (L; mock-infected) or heavy formaldehyde ( $\mathrm{H}$; CrPV-infected). Peptides were then pooled and analyzed by LC-MS/MS. (B) and (C) $\log _{2}$ abundance ratio distribution of proteins quantified from CrPV- and mock-infected cells for the (B) cell pellets and the (C) ELVs. (D) Scatter plot of proteins quantified in S2 cell pellets in comparison to those in ELVs. Plotted is the $\log _{2}$ transformed abundance ratios comparing the heavy (CrPV-infected) to light (mock) labeled samples (H/L ratio). Boxed region is zoomed-in area of the graph lacking viral proteins.

\section{Discussion}

Viruses have evolved novel strategies to evade the host immune system. In recent years, it has been demonstrated that non-enveloped viruses have the ability to co-opt cellular processes to acquire an envelope ${ }^{32,33}$. In this study, we provide evidence that CrPV structural proteins and viral RNA are enveloped within ELVs derived from CrPV-infected S2 cells and that these ELVs are infectious. By using proteomic approaches, we identified 1408 and 802 proteins from the cell and exosome-like vesicles, respectively (Fig. 3). Only 64\% of the ELV-associated proteins overlapped with the cell pellet suggesting that there may be a distinct set of proteins enriched within ELVs compared to the cell pellet. Moreover, during CrPV infection, 40 proteins increased and 8 protein decreased in abundance compared to mock-infected cells (Fig. 3), thus suggesting an active mechanism for sorting and secretion of proteins in infected-S2 cells. We propose that CrPV uses both lytic and non-lytic egress strategies as part of its viral life cycle. 

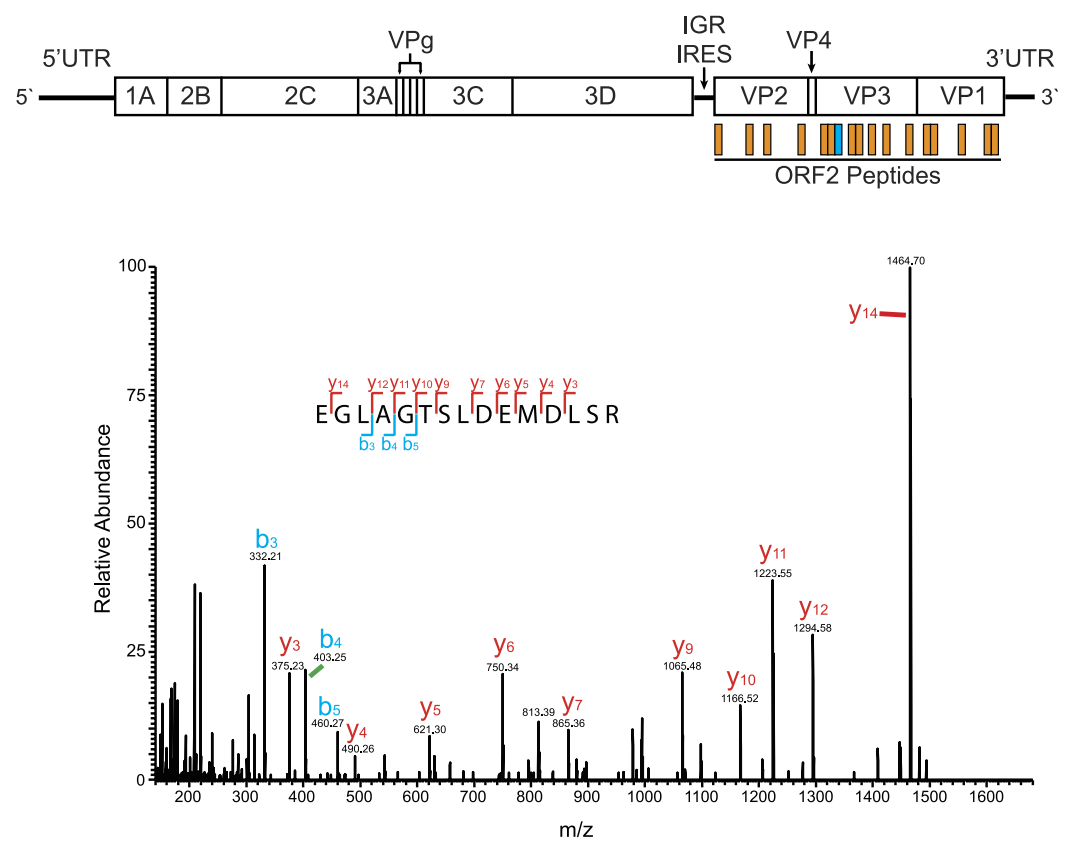

Figure 4. CrPV ORF2 peptides identified in ELVs from CrPV-infected S2 cells. Top: schematic displaying 21 peptides mapped to CrPV ORF2 (note: does not include peptides with missed cleavages). Bottom: MS/ MS spectra of one peptide identified (blue in schematic) from the CrPV ORF2 enriched in ELVs derived from CrPV-infected cells. Fragment spectra from the $813.40 \mathrm{~m} / \mathrm{z}$ doubly charged, formaldehyde labeled $(+32 \mathrm{Da})$ precursor ion of EGLAGTSLDEMDLSR from trypsin digested ELVs. In total, 21 peptides were mapped to CrPV ORF2. Blue bar indicates location of displayed peptide. Individual fragment ions are annotated in the spectrum and in the sequence representation. In red are y-ions, while b-ions are represented in blue.

The best-described mechanism for the formation of exosomes in mammalian cells is through the endosomal sorting complex required for transport (ESCRT) $)^{27}$. This complex is composed of roughly thirty proteins that assemble into four complexes (ESCRT-0, -I, -II, and -III) along with other associated proteins. ESCRT-0 binds and sequesters ubiquitylated transmembrane proteins in the endosomal membrane. HRS (a component of ESCRT-0) then recruits TSG101 of the ESCRT-I complex. In tandem with ESCRT-II, ESCRT-I acts to cause endosomal membrane deformation. ESCRT-I is then involved in the recruitment of ESCRT-III via ESCRT-II or ALIX. Finally, ESCRT-III facilitates vesicle scission into the endosomal lumen ${ }^{27,34}$. The exact mechanism of how cytosolic proteins are sorted into exosomes is not well understood. It is thought that mono-ubquitinylated proteins containing 'late' domain with a sequence motif $\mathrm{P}(\mathrm{S} / \mathrm{T}) \mathrm{XP}, \mathrm{YPX}_{1-3} \mathrm{~L}$, or PPXYY are targeted for delivery to MVBs by interacting with proteins such as TSG101 or ALIX ${ }^{26,32,35}$. Alternatively, a recent role for HSC70 has been proposed where it binds proteins containing a KFERQ sequence and to phosphatidylserine on the MVB outer membrane ${ }^{36}$. However, not all proteins in exosomes are ubquitylated nor do they all contain KFERQ sequences. It appears a passive mechanism may be involved in protein sorting to MVBs that involves lipid (e.g. ceramide or cholesterol) and/or tetraspannin associations ${ }^{37-40}$. Currently there is limited knowledge on how CrPV alters host cell processes during infection and although numerous other viruses have been shown to exploit exosome biogenesis pathway including human immunodeficiency virus-1 (HIV-1), hepatitis B virus (HBV), and Epstein Barr virus (EBV), there remains no consensus view of a distinct mechanism that leads to changes in cargo content of exosomes ${ }^{41}$.

From our proteomic analysis, the only protein to be enriched outside of viral proteins in the cell pellet was CG31731-RC, also recently termed Eato ${ }^{42}$. This protein is homologous to the C. elegans CED-7 and mammalian ABCA1 proteins and has recently been shown to be important for the cellular engulfment pathway in Drosophila ovaries $^{42}$. In mouse hemocytes and thymocytes, ABCA1 has a role in presentation of phosphatidylserine after apoptotic stimuli, and thus may act in dying cells to promote phagocytic engulfment ${ }^{43}$. Moreover, CED-7 in C. elegans has been shown to be important for the presence of extracellular vesicles containing engulfment signals and its activity is required in both phagocytic and dying cells for engulfment ${ }^{44,45}$. Given that phagocytosis plays an important role in Drosophila innate immunity ${ }^{46}$, upregulation of CG31731/Eato may act as first line defense against viral infection to clear infected cells. Alternatively, as discussed below, this protein may be important in secretion of anti-viral vesicles involved in the RNAi response ${ }^{16}$. Further study of this protein and its role during infection will likely provide insight into the host-virus interactions occurring in Drosophilia.

Surprisingly, the most highly enriched protein(s) in ELVs derived from CrPV-infected cells was the CrPV structural proteins. We identified 21 peptides that mapped across the CrPV ORF2 polyprotein resulting in a $\log _{2}$ fold change of $>12$ in ELVs (Figs 3 and 4). Interestingly, we were unable to identify any of the CrPV non-structural proteins encoded by ORF1 either by mass spectrometry or Western blot within the ELV fraction (Figs 2 and 3). Together with our density gradient analysis, viral titres, and membrane integrity assay (Figs 5 and 6), these results indicate that, like hepatitis A virus (HAV) and hepatitis E virus (HEV), CrPV may co-opt 


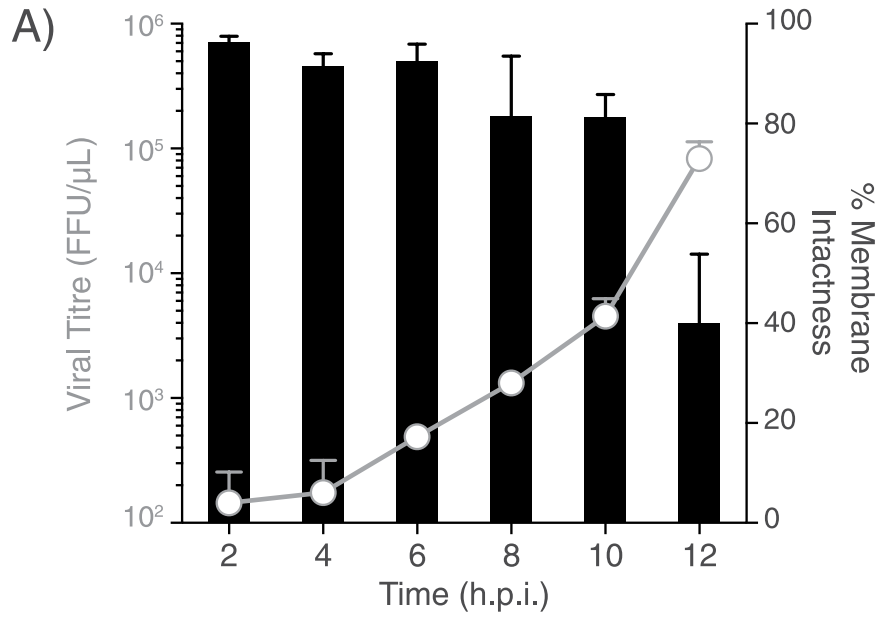

B)
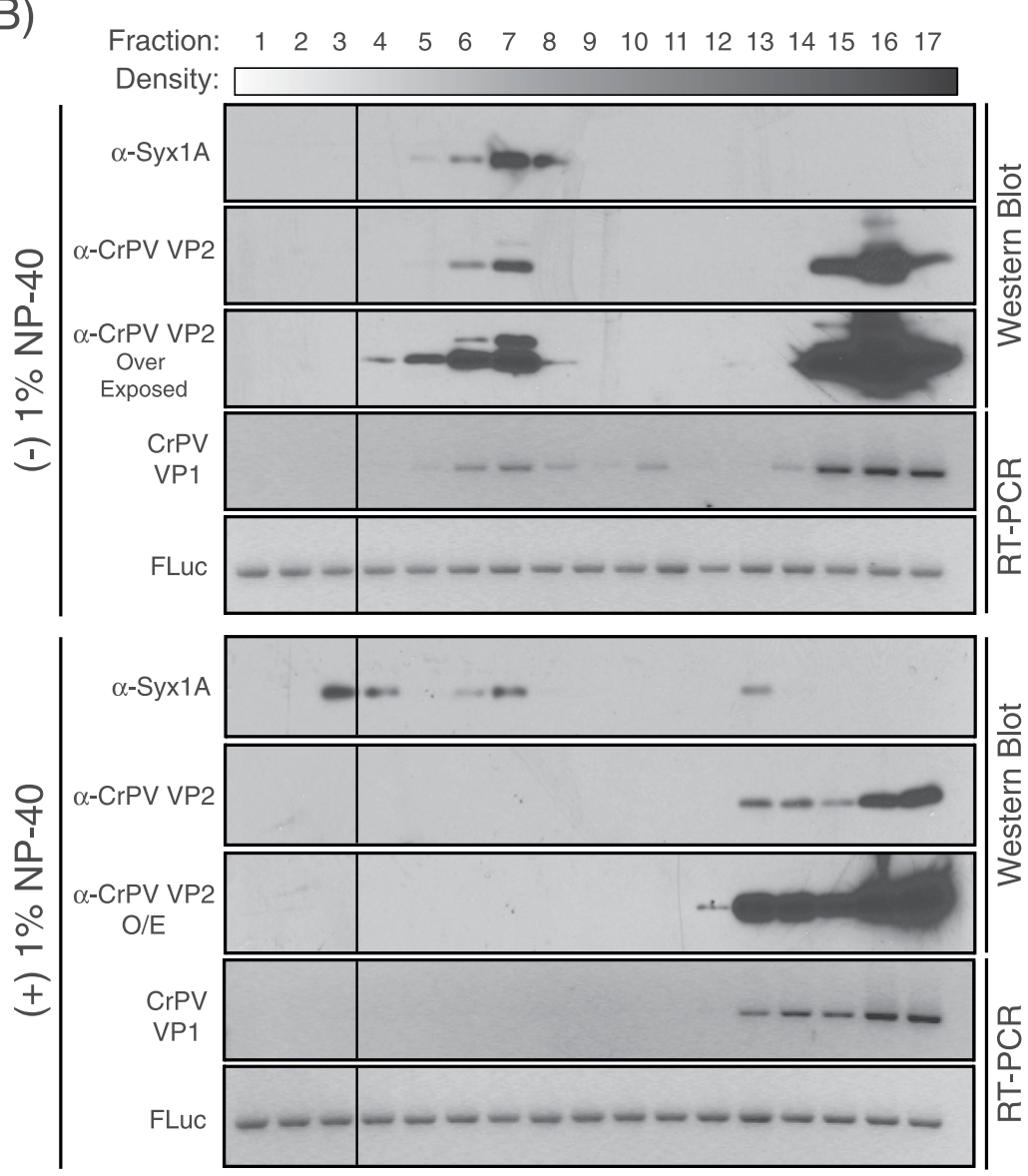

Figure 5. ELVs from CrPV-infected $\mathrm{S} 2$ cells contain CrPV RNA and structural protein. (A) Membrane integrity and extracellular viral yield in CrPV-infected S2 cells. S2 cells were infected with CrPV at an MOI of 10 and viral loads were measured in the supernatant at various time points throughout infection via a fluorescence foci forming assay (open circles). Exclusion of trypan blue staining of S2 cells (i.e. percent cell viability) was used as a proxy for membrane integrity at each time point (bar graph). Shown is the average from three biological replicates $( \pm S D)$. (B) Iodixanol gradient analysis of ELVs from mock- and CrPV-infected S2 cells. ELVs from CrPV-infected S2 cells (MOI 10, $24 \mathrm{hpi}$ ) were treated with or without $1 \%$ NP-40 and layered onto an 8-40\% iodixanol gradient and centrifuged at $141,000 \mathrm{~g}$ for 48 hours. Fractions were collected, split, and protein was extracted via trichloroacetic acid precipitation and RNA via phenol-chloroform. Syx-1A was used as a marker for ELVs. Equal amounts of In vitro synthesized firefly luciferase RNA was doped into each fraction to control for RT-PCR efficiency. Shown is a representative gel from three independent experiments. Uncropped gel images are shown in Supplemental Fig. S5.

exosomes for its own advantage during infection ${ }^{32}$. Akin to our observations with CrPV, HAV infection leads to two distinct virus populations of virus; both populations remain infectious, however one acquires an envelope (eHAV) while the other remains non-enveloped ${ }^{5}$. HAV encodes two tandem 'late' domains in its VP2 capsid 


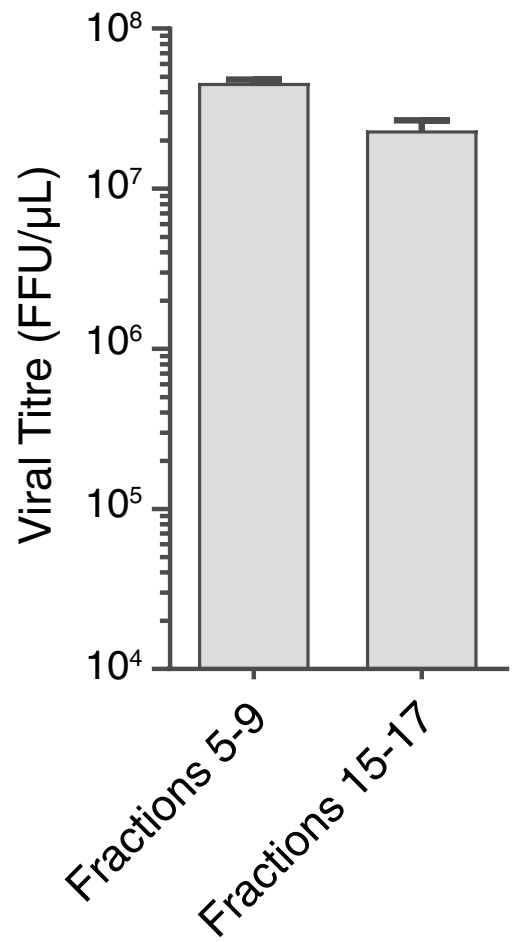

Figure 6. ELV s from infected S2 cells are infectious. ELVs from CrPV-infected cells (24 hpi) were isolated and subjected to iodixanol gradients analysis. Fractions 5-9 (ELVs) and 15-17 (virions) were collected, pooled, and added to naïve S2 cells. After 48 hours, cells were lysed and viral titres were determined using a fluorescence foci forming assay. Shown is the average from three biological replicates $( \pm S D)$.

protein that are proposed to facilitate interaction of the viral capsid with ALIX and be subsequently sorted into exosomes. Intriguingly, eHAV contains an unprocessed version of the viral capsid protein VP1, termed VP1pX, that is not present in the non-enveloped virus suggesting there could an additional role played by the $\mathrm{pX}$ extension to facilitate envelopment of $\mathrm{HAV}^{5}$. Similarly, HEV also contains a viral protein (ORF3) that is only found in enveloped forms of the virus which directs interacts with TSG101 through a PSAP motif ${ }^{47,48}$. Whether CrPV follows a similar mechanism(s) remains to be determined. Further studies investigating the role of the ESCRT complex and how viral proteins contribute to this process in $\mathrm{CrPV}$ infection would provide valuable insights into its mode of transmission.

Why would CrPV require an envelope for cell-to-cell transmission? With HAV, enveloped virions circulate in the blood of infected patients while non-enveloped virions are found in the feces ${ }^{5}$. For decades virologists have understood that enveloped and non-enveloped viruses have distinct advantages over on another. Enveloped viruses can readily manipulate the contents of their cognate envelope allowing for flexibility in receptors, evasion of the host immune response, and shuttling viral proteins to enhance infection. Nevertheless, envelopes are typically susceptible to bile salts and retain lower stability in the environment (e.g. due to desiccation or chemical resistance $)^{49}$. Thus, a biphasic viral life cycle provides the security of an envelope with the robustness of a capsid. In the case of eHAV, the envelope cloaks the virus from neutralizing antibodies in the bloodstream. Unlike humans, Drosophila lacks an adaptive immune system; however, hemocytes circulating in the hemolymph mediate phagocytosis of invading pathogens to prevent infection ${ }^{46}$. Furthermore, Drosophilia cells secrete the Ig-domain containing protein, Dscam, which can be expressed as over 18,000 isoforms and may act in opsonizing pathogens for phagocytosis ${ }^{46,50}$. It is plausible that CrPV hijacks the exosomal pathway to shroud itself from the phagocytic machinery within the host organism. Finally, insect ELVs are not only pro-viral; ELVs secreted from hemocytes of virally-infected fruit flies contain virally-encoded siRNAs to elicit a systemic RNAi immune response ${ }^{16}$. It will be important to determine the mechanisms by which host and viral determinants govern provs anti-viral ELVs and whether the ELVs are more infectious than non-enveloped CrPV.

Overall, our data offers the first look into how extracellular vesicles contribute to the pathogenesis of CrPV and how these viruses are transmitted between host cells. Moreover, it suggests that this virus can co-opt these vesicles for its own advantage to potentially evade the host immune response. Whether other members of dicistroviruses utilize ELVs and how this process is regulated during dicistrovirus infection warrants further investigation.

\section{References}

1. Flint, S. J. American Society for Microbiology. Principles of virology. 3rd ed. (ASM Press, Washington, DC 2009)

2. Vaheri, A. et al. Uncovering the mysteries of hantavirus infections. Nature reviews. Microbiology 11, 539-550 (2013).

3. Romero-Brey, I. et al. Three-dimensional architecture and biogenesis of membrane structures associated with hepatitis $\mathrm{C}$ virus replication. PLoS Pathog 8, e1003056 (2012). 
4. Rossman, J. S. \& Lamb, R. A. Influenza virus assembly and budding. Virology 411, 229-236 (2011).

5. Feng, Z. et al. A pathogenic picornavirus acquires an envelope by hijacking cellular membranes. Nature 496, 367-371 (2013).

6. Chen, Y. H. et al. Phosphatidylserine vesicles enable efficient en bloc transmission of enteroviruses. Cell 160, 619-630 (2015).

7. Bird, S. W., Maynard, N. D., Covert, M. W. \& Kirkegaard, K. Nonlytic viral spread enhanced by autophagy components. Proc Natl Acad Sci USA 111, 13081-13086 (2014).

8. Takahashi, M. et al. Hepatitis E Virus (HEV) strains in serum samples can replicate efficiently in cultured cells despite the coexistence of HEV antibodies: characterization of HEV virions in blood circulation. J Clin Microbiol 48, 1112-1125 (2010).

9. Thery, C., Zitvogel, L. \& Amigorena, S. Exosomes: composition, biogenesis and function. Nature reviews. Immunology 2, 569-579 (2002).

10. Raposo, G. \& Stoorvogel, W. Extracellular vesicles: exosomes, microvesicles, and friends. J Cell Biol 200, 373-383 (2013).

11. Meckes, D. G. Jr. \& Raab-Traub, N. Microvesicles and viral infection. J Virol 85, 12844-12854 (2011)

12. Robbins, P. D. \& Morelli, A. E. Regulation of immune responses by extracellular vesicles. Nature reviews. Immunology 14, 195-208 (2014).

13. Koppen, T. et al. Proteomics analyses of microvesicles released by Drosophila Kc167 and S2 cells. Proteomics 11, 4397-4410 (2011).

14. Wang, T., Fang, L., Zhao, F., Wang, D. \& Xiao, S. Exosomes Mediate Intercellular Transmission of Porcine Reproductive and Respiratory Syndrome Virus. J Virol, 92 (2018)

15. Wei, T., Hibino, H. \& Omura, T. Release of Rice dwarf virus from insect vector cells involves secretory exosomes derived from multivesicular bodies. Communicative \& integrative biology 2, 324-326 (2009).

16. Tassetto, M., Kunitomi, M. \& Andino, R. Circulating Immune Cells Mediate a Systemic RNAi-Based Adaptive Antiviral Response in Drosophila. Cell 169, 314-325 e313 (2017).

17. Kerr, C. H. \& Jan, E. Commandeering the Ribosome: Lessons Learned from Dicistroviruses about Translation. J Virol 90, 5538-5540 (2016).

18. Bonning, B. C. \& Miller, W. A. Dicistroviruses. Annu Rev Entomol 55, 129-150 (2010).

19. Garrey, J. L., Lee, Y. Y., Au, H. H., Bushell, M. \& Jan, E. Host and viral translational mechanisms during cricket paralysis virus infection. J Virol 84, 1124-1138 (2010).

20. Boersema, P. J., Aye, T. T., van Veen, T. A., Heck, A. J. \& Mohammed, S. Triplex protein quantification based on stable isotope labeling by peptide dimethylation applied to cell and tissue lysates. Proteomics 8, 4624-4632 (2008).

21. Cox, J. \& Mann, M. MaxQuant enables high peptide identification rates, individualized p.p.b.-range mass accuracies and proteomewide protein quantification. Nature biotechnology 26, 1367-1372 (2008)

22. Tyanova, S., Temu, T. \& Cox, J. The MaxQuant computational platform for mass spectrometry-based shotgun proteomics. Nature protocols 11, 2301-2319 (2016).

23. Tyanova, S. et al. The Perseus computational platform for comprehensive analysis of (prote)omics data. Nature methods 13, 731-740 (2016).

24. Gillis, J., Mistry, M. \& Pavlidis, P. Gene function analysis in complex data sets using Ermine. J. Nature protocols 5, 1148-1159 (2010).

25. Beckett, K. et al. Drosophila S2 cells secrete wingless on exosome-like vesicles but the wingless gradient forms independently of exosomes. Traffic 14, 82-96 (2013).

26. Buschow, S. I., Liefhebber, J. M., Wubbolts, R. \& Stoorvogel, W. Exosomes contain ubiquitinated proteins. Blood Cells Mol Dis 35, 398-403 (2005).

27. Colombo, M., Raposo, G. \& Thery, C. Biogenesis, secretion, and intercellular interactions of exosomes and other extracellular vesicles. Annu Rev Cell Dev Biol 30, 255-289 (2014).

28. Scotti, P. D., Longworth, J. F., Plus, N., Croizier, G. \& Reinganum, C. In Lauffer, M. A., Bang, F. B., Maramorosch, K. and Smith, K. M. (eds), Advances in virus research. Academic Press, New York, Vol. 26, pp. 118-144 (1981).

29. Kerr, C. H. et al. The $5^{\prime}$ untranslated region of a novel infectious molecular clone of the dicistrovirus cricket paralysis virus modulates infection. J Virol 89, 5919-5934 (2015).

30. Wilson, J. E., Powell, M. J., Hoover, S. E. \& Sarnow, P. Naturally occurring dicistronic cricket paralysis virus RNA is regulated by two internal ribosome entry sites. Mol Cell Biol 20, 4990-4999 (2000).

31. Cantin, R., Diou, J., Belanger, D., Tremblay, A. M. \& Gilbert, C. Discrimination between exosomes and HIV-1: purification of both vesicles from cell-free supernatants. J Immunol Methods 338, 21-30 (2008).

32. Feng, Z. \& Lemon, S. M. Peek-a-boo: membrane hijacking and the pathogenesis of viral hepatitis. Trends in microbiology 22, 59-64 (2014).

33. Bird, S. W. \& Kirkegaard, K. Escape of non-enveloped virus from intact cells. Virology 479-480, 444-449 (2015).

34. Hurley, J. H. \& Hanson, P. I. Membrane budding and scission by the ESCRT machinery: it's all in the neck. Nature reviews. Molecular cell biology 11, 556-566 (2010).

35. Freed, E. O. Viral late domains. J Virol 76, 4679-4687 (2002).

36. Sahu, R. et al. Microautophagy of cytosolic proteins by late endosomes. Dev Cell 20, 131-139 (2011).

37. Trajkovic, K. et al. Ceramide triggers budding of exosome vesicles into multivesicular endosomes. Science 319, 1244-1247 (2008).

38. van Niel, G. et al. The tetraspanin CD63 regulates ESCRT-independent and -dependent endosomal sorting during melanogenesis. Dev Cell 21, 708-721 (2011).

39. Edgar, J. R., Eden, E. R. \& Futter, C. E. Hrs- and CD63-dependent competing mechanisms make different sized endosomal intraluminal vesicles. Traffic 15, 197-211 (2014).

40. Stuffers, S., Sem Wegner, C., Stenmark, H. \& Brech, A. Multivesicular endosome biogenesis in the absence of ESCRTs. Traffic 10, 925-937 (2009).

41. Raab-Traub, N. \& Dittmer, D. P. Viral effects on the content and function of extracellular vesicles. Nature reviews. Microbiology 15, 559-572 (2017).

42. Santoso, C. S. et al. The ABC Transporter Eato Promotes Cell Clearance in the Drosophila melanogaster Ovary. G3 (Bethesda) 8 , 833-843 (2018).

43. Hamon, Y. et al. $\mathrm{ABC} 1$ promotes engulfment of apoptotic cells and transbilayer redistribution of phosphatidylserine. Nat Cell Biol 2, $399-406(2000)$

44. Wu, Y. C. \& Horvitz, H. R. The C. elegans cell corpse engulfment gene ced-7 encodes a protein similar to ABC transporters. Cell 93, 951-960 (1998).

45. Mapes, J. et al. CED-1, CED-7, and TTR-52 regulate surface phosphatidylserine expression on apoptotic and phagocytic cells. Curr Biol 22, 1267-1275 (2012)

46. Stuart, L. M. \& Ezekowitz, R. A. Phagocytosis and comparative innate immunity: learning on the fly. Nature reviews. Immunology 8 , 131-141 (2008).

47. Nagashima, S. et al. A PSAP motif in the ORF3 protein of hepatitis E virus is necessary for virion release from infected cells. J Gen Virol 92, 269-278 (2011).

48. Nagashima, S. et al. Tumour susceptibility gene 101 and the vacuolar protein sorting pathway are required for the release of hepatitis E virions. J Gen Virol 92, 2838-2848 (2011).

49. Smith, W. The action of bile salts on viruses. J Pathol Bacteriol 48, 557-571 (1939).

50. Watson, F. L. et al. Extensive diversity of Ig-superfamily proteins in the immune system of insects. Science 309, 1874-1878 (2005). 


\section{Acknowledgements}

We thank both Foster and Jan labs for discussions. The study was supported by a CIHR Projects Grant (PJT148761) to E.J., a CIHR operating grant (MOP-776888) to L.F. and an NSERC CGS D Fellowship to C.K.

\section{Author Contributions}

C.K., L.F. and E.J. wrote the main manuscript text and prepared all figures. All authors reviewed the manuscript. C.K., L.F. and E.J.: Conception and design, data analysis and interpretation, manuscript preparation. C.K., U.D. and N.S.: Collection and assembly of data. C.Y., L.F. and E.J.: Administrative and financial support. All authors read and approved the manuscript.

\section{Additional Information}

Supplementary information accompanies this paper at https://doi.org/10.1038/s41598-018-35717-5.

Competing Interests: The authors declare no competing interests.

Publisher's note: Springer Nature remains neutral with regard to jurisdictional claims in published maps and institutional affiliations.

(c) (i) Open Access This article is licensed under a Creative Commons Attribution 4.0 International License, which permits use, sharing, adaptation, distribution and reproduction in any medium or format, as long as you give appropriate credit to the original author(s) and the source, provide a link to the Creative Commons license, and indicate if changes were made. The images or other third party material in this article are included in the article's Creative Commons license, unless indicated otherwise in a credit line to the material. If material is not included in the article's Creative Commons license and your intended use is not permitted by statutory regulation or exceeds the permitted use, you will need to obtain permission directly from the copyright holder. To view a copy of this license, visit http://creativecommons.org/licenses/by/4.0/.

(C) The Author(s) 2018 